\title{
Uma Investigação Empírica Sobre a Curva de Depreciação de Valores de Imóveis Utilizando Inferência Estatística
}

\section{Marco Aurélio Stumpf Gonzalez}

Engenheiro Civil, Mestre em Engenharia

Doutorando em Engenharia - NORIE/UFRGS (Universidade Federal do

Rio Grande do Sul/RS)

Professor da UNISINOS (Universidade do Vale do Rio dos Sinos, São Leopoldo/RS)

gonzalez@prisma.unisinos.tche.br / http://www.unisinos.tche.br

Rua Dr. Eduardo Chartier,211/304 - (051) 342.3617 - Porto Alegre/RS

$-90520-000$

Palavras-chave: Avaliação de imóveis; Depreciação; Mercado imobiliário; Inferência estatística; Modelos hedônicos.

Key words: real estate values; depreciation; real estate market; statistical inference; hedonic models.

\section{RESUMO}

Os imóveis possuem grande durabilidade, e no decurso de sua vida útil, sofrem diminuições de qualidade, devidas ao uso e à desatualização funcional. Estas perdas levam à progressiva redução do valor que o imóvel atinge no mercado. $O$ formato desta curva de desvalorização não é conhecido, à principio. Este artigo apresenta as técnicas de estimação da depreciação para um conjunto de dados. Os modelos foram encontrados por regressão múltipla, comparando-se os resultados com os obtidos empregando os coeficientes padronizados (arbitrários), na forma tradicional.

\section{ABSTRACT}

The real estate goods has great durability, and its quality progressively reduces about the economic life, by functional and use. This losses goes to diminishing market value. The functional form of this curve isn t know. This paper presents the estimation techniques to depreciation, with regression analysis, comparing with the traditional (and arbitrary) coefficients. 


\section{PRODUÇÃO}

\section{Introdução}

Os imóveis são bens compostos, existindo múltiplas características de interesse, e a comparação direta não é possível. Existem vários métodos para a determinação do valor de um imóvel. $O$ melhor procedimento é a comparação de dados de mercado, através da ponderação de seus vários atributos, com os pesos obtidos em equações de regressão, inferindo-se o valor desejado através da substituição das características do imóvel no modelo. É uma tarefa delicada, que depende muito da qualidade dos dados e do respeito aos pressupostos básicos da análise estatística

Embora seja inelutavelmente superior como técnica, a inferência ainda não é adotada por todos os profissionais que militam no ramo. Além de exigir uma razoável quantidade de dados para os modelos (de regra, quatro vezes o número de parâmetros da equação a ser estimada), demanda sólidos conhecimentos de inferência estatística, os quais geralmente não são obtidos nas universidades. Além disto, a matemática envolvida é forte, envolvendo inversões de matrizes e numerosos somatórios. Há alguns anos, este problema inviabilizava a utilização da inferência nas avaliações rotineiras. Esta barreira, tecnológica, foi vencida há mais de uma década: os computadores pessoais permitem a realização rápida dos cálculos necessários, existindo hoje vários softwares aplicados à avaliação de imóveis (González, 1997).
A avaliação de imóveis através de modelos pré-determinados ou pelo custo de reprodução é bem mais simples e ainda é muito usada, principalmente em plantas de valores. $O$ processo de homogeneização de fatores é inadequado, por várias razões, mas principalmente por não considerar as informações obtidas diretamente do mercado imobiliário, com as correções ficando ao arbitrio do avaliador. Problemas semelhantes são enfrentados na avaliação pelo custo de reprodução.

Os valores obtidos por uma ou outra técnica podem divergir assustadoramente. Entretanto, formalmente todos são estimativas do verdadeiro valor de mercado, ficção que só é alcançada sob condições ideais. Em termos extremamente simplificados, pode-se dizer que o valor de mercado seria obtido sob concorrência perfeita. Contudo, sabese que o mercado imobiliário não é de concorrência perfeita, pois não há informação adequada ou igualdade entre os agentes, por exemplo (González, 1997a). A análise é complexa, porque os métodos tradicionais, embora permitidos pela Norma de avaliações nos níveis inferiores de qualidade, contém forte dose de arbítrio (ABNT, 1989). Na prática profissional, a comparação de avaliações obtidas por métodos diferentes é necessária freqüentemente. Em procedimentos judiciais, por exemplo, é comum serem apresentados laudos divergentes, problema que precisa ser resolvido no âmbito da técnica. Gilson Lima (1995) abordou com propriedade o assunto, indicando a inferência como 


\section{PRODUÇÃO}

caminho para a verificação de modelos de homogeneização, obtendo-se os "fatores de correção" pela metodologia científica.

Como uma contribuição ao estudo, apresenta-se a seguir uma comparação de cálculo da depreciação dos imóveis através de inferência e pelas fórmulas tradicionais, demonstrando o processo de obtenção dos coeficientes, que pode ser posteriormente generalizado para os outros atributos. Diversos modelos são apresentados, optando-se pelos de melhor desempenho nos testes estatísticos. Foi utilizado um conjuntos de dados de apartamentos residenciais em Porto Alegre. Os modelos obtidos são comparados com as fórmulas correntes para cálculo da depreciação.

Esta é uma análise simplificada. Podese argumentar que o valor total do imóvel não poderia ser empregado para detectar a depreciação, porque esta age apenas sobre a construção. Seria necessário separar o valor correspondente ao terreno (no caso de apartamentos, à fração ideal). Obtendo-se uma amostra de terrenos na mesma área, o valor do terreno pode ser obtido por inferência estatística, subtraindo-se então esta parcela do valor total (esta operação é uma avaliação do valor da construção pelo método residual). Assim os residuos, correspondentes à construção em si, seriam empregados na estimação. Contudo, pode-se pensar também que o valor dos terrenos vagos é diferente do valor dos terrenos ocupados, ou seja, a construção não poderia ser obtida diretamente pela subtração, mesmo admitindo-se que as possibilidades de construção tenham permanecido, desde a construção do edificio, assim como as características de entorno.

\section{O mercado imobiliário}

O mercado imobiliário é um dos setores mais complexos da economia, sendo influenciado por inúmeros fatores, locais ou globais. Existem alguns elementos macro-econômicos que conferem um comportamento nacional, por assim dizer, ao mercado imobiliário, tais como inflação, confiança na condução da economia, oferta de crédito para construção e taxa de juros. Estas influências são gerais, afetando toda uma região, e seus efeitos não são detectados nas análises de valores de mercado, as quais consistem essencialmente de comparações micro-econômicas entre unidades semelhantes, próximas fisica e temporalmente (Balarine, 1996; Franchi, 1991; González, 1993; Lucena, 1985).

Porém, de outra parte, as variações intra-urbanas provocam constantes alteraçס̃es nos preços praticados no mercado. Existem diferenças de uma região para outra, dentro da mesma cidade. Em função da atuação dinâmica $e$ não coordenada dos agentes, simultaneamente existem áreas que estão sendo valorizadas e outras que estão sendo desvalorizadas. O capital imobiliário age alterando a distribuição relativa destas áreas, transformando os padrões de uso de cada região como forma de alcançar 


\section{PRODUÇÃO}

seu lucro, basicamente fundiário. Inicialmente, os terrenos são valorizados pela disputa entre as incorporadoras. Contudo, o surgimento de novas construções provoca perturbações também nos preços dos imóveis usados do entorno. A valorização ocorre ainda por efeito de grandes empreendimentos, como shopping centers, ou pela revitalização de uma área que anteriormente não recebia investimentos públicos, por exemplo. Já as desvalorizações podem surgir pela instalação de atividades poluidoras, dificuldades de transporte ou surgimento de sub-habitações. Como todas estas externalidades não são homogeneamente distribuídas, os preços são pressionados desigualmente (Abramo, 1988; Dubin, 1992; Maraschin, 1993).

Uma das propriedades especiais dos imóveis é a grande vida útil. A medição da influência desta característica nos preços é realizada através da idade do imóvel. A depreciação, funcional ou de uso, afeta significativamente o valor de mercado e está ligada ao estado de conservação e ao estilo do imóvel. Adotando-se a inferência estatística, é preciso simplesmente incluir a variável no modelo, obtendo-se o coeficiente correspondente pelo processamento estatístico, com o valor e a importância do coeficiente sendo garantida pelos testes estatísticos aplicados sobre as variáveis e sobre os modelos (Cuthbertson et alii, 1992; González, 1997). Para aplicar os métodos de homogeneização ou custo de reprodução, é necessário considerar a depreciação da construção através de fórmulas genéricas. As funções aplicadas atualmente para a consideração das depreciações podem ser encontradas em vários textos, como os de Fiker (1993), IBAPE (1974), Lopes (1995), McMichael (1962) e Moreira (1994). São idéias tradicionais de depreciação segundo uma função especial (reta, parábola ou outra qualquer), escolhida de acordo com as preferências do avaliador, sem considerar as particularidades do mercado.

Porém, diante da dinâmica intraurbana, não se pode simplesmente aceitar a hipótese de uma única função de depreciação para a cidade inteira, e menos ainda para qualquer cidade indistintamente. O perfil de depreciação dos imóveis pode variar no tempo e no espaço e é preciso verificar empiricamente, em cada caso, qual a curva real. A racionalidade do procedimento está em buscar imóveis semelhantes ao avaliando e, através da comparação de várias equações de regressão alternativas, escolher a melhor função de depreciação, através do desempenho nos testes estatísticos.

\section{Cálculo da depreciação através de fórmulas}

A depreciação está relacionada com a conservação do imóvel. O uso e a manutenção influem na parcela de valor perdida pela construção, em um determinado momento de sua vida útil. A avaliação do estado de conservação é uma tarefa dificil, dependendo do julgamento de cada profissional. Geralmente a idade 


\section{PRODUÇÃO}

do imóvel é empregada para os cálculos. Pode-se usar a idade fiscal (contada à partir do "Habite-se") ou a idade aparente (resultado da inspeção e julgamento do profissional). Nas fórmulas, elas são cotejadas contra a vida útil provável. Tanto idade fiscal quanto aparente contém um inconveniente estatístico, que é a consideração de uma escala discreta. Por exemplo, um imóvel vistoriado em dezembro e outro em janeiro terão um ano de diferença na data cadastrada pela municipalidade, quando deveriam ter apenas um mês.

A análise de uma amostra de grandes dimensões diminui este problema, embora impeça o uso de vistorias ou inspeções in loco. A idade fiscal é empregada corriqueiramente, na literatura de economia urbana e nas avaliações de imóveis, como proxy para estado de conservação, admitindo-se implicitamente que há proporcionalidade entre idade $\mathrm{e}$ condição do imóvel.

Existem inúmeras fórmulas para cálculo da depreciação, algumas bastante imaginativas. Esta diversidade deve-se, em parte, ao desconhecimento da verdadeira função. Assim, quando as fórmulas comuns não se ajustam, o profissional sente-se tentado a encontrar uma fórmula "melhor" ou mais apropriada aos dados que possui. Apenas os métodos mais conhecidos são apresentados aqui (Fiker, 1993; Moreira, 1994):

a) Método da linha reta: $\mathrm{Va}=\mathrm{Vr}+$
$\mathrm{Vd} *(\mathrm{U}-\mathrm{I}) / \mathrm{U}$

b) Método da parábola (Kuentzle): $\mathrm{Va}=\mathrm{Vn}-\mathrm{Vd}^{*}(\mathrm{U} / \mathrm{I})^{2}$

c) Método de Ross: $\mathrm{Va}=\mathrm{Vn}-\mathrm{Vd}^{*}$ $\left(\mathrm{I} / \mathrm{U}+\mathrm{I}^{2} / \mathrm{U}^{2}\right) / 2$

Onde as variáveis empregadas são:

*Va: valor atual (após o desconto da depreciação);

*Vn: valor do imóvel novo;

*Vd: valor depreciável (parcela que pode sofrer depreciação);

*Vr: valor residual (de demolição, não depreciável);

*I: idade fisica (fiscal) do imóvel;

*U: vida útil;

Nestes métodos, existe um valor mínimo, residual, que não é afetado (Vr). Geralmente esta parcela é considerada como $20 \%$ do valor total do imóvel novo (Vn). A parcela que sofre depreciação (Vd) seria de $80 \%$ do total, portanto. A vida útil (U) indica o limite de utilização econômica do imóvel. Para apartamentos e residências unifamiliares, geralmente é considerada como 60/70 anos, embora o mercado imobiliário apresente evidências de que $o$ uso se extende bem mais que isto. Em qualquer um destes métodos, a idade fisica ou fiscal pode ser substituida pela idade aparente, obtida em vistorias detalhadas dos imóveis.

Devem ser citados ainda os métodos de Heidecke, que emprega um quadro relacionando a classificação da conservação do imóvel (de "ótimo" à "demolição") a um coeficiente de depreciação (de $0 \%$ a 100\%), e o de RossHeidecke, que combina os dois métodos, 


\section{PRODUÇÃO}

gerando uma tabela de coeficientes de depreciação, obtidos pela consideração simultânea da parcela Idade/Vida Útil e do estado de conservação (IBAPE, 1974; Moreira, 1995).

Diante da variedade de critérios, a questão principal é a escolha do método a ser empregado. Não há certeza de qual deles seja o mais adequado, nem de que um deles realmente se aplique ao caso específico. Uma alternativa, proposta neste trabalho, é inferir a curva real para as condições do mercado em análise, usando-a como base para escolher o método ou para adaptá-lo às necessidades. Isto pode ser realizado através da análise de regressão, testando estatisticamente os modelos. A possibilidade de testar o desempenho é importante, pois elimina a incerteza, criando uma base científica de escolha dos métodos.

\section{Cálculo da depreciação através de análise empírica}

A investigação da curva de melhor ajuste foi realizada através da montagem de modelos hedônicos de preços, relacionando as variáveis coletadas. Foram escolhidos alguns formatos para a variável que mede o tempo (Idade), para a comparação com as fórmulas apresentadas. $O$ ajuste das curvas foi realizado com regressão múltipla, pelo método dos mínimos quadrados ordinários $(O L S)$, geralmente empregados com esta finalidade.
Nesta etapa de investigação empírica, foi utilizada uma amostra de apartamentos residenciais transacionados em Porto Alegre no período de Janeiro de 1990 a Junho de 1994. Os dados originam-se de declarações para tributação pelo imposto de transmissão (ITBI), obtidas junto à Secretaria da Fazenda do Municipio. Detalhes sobre os dados podem ser obtidos em González (1996). Para este trabalho, foram selecionados 1030 imóveis e os valores declarados foram corrigidos monetariamente até Janeiro de 1996 através do IGP-DI (Fundação Getúlio Vargas). As variáveis estão relacionadas na Tabela 1, a seguir.

Inicialmente, foram compilados modelos hedônicos nos dois formatos básicos mais comuns, com a variável dependente (Valor), nas formas linear e semi-logarítmica, empregando a variável Idade na forma linear. A seleção das demais variáveis foi feita pelo método stepwise. Os modelos apresentaram resultados semelhantes nas duas formas, optando-se pela linear pela interpretação dos coeficientes.

Com este modelo básico, variando apenas a forma da variável Idade, foram testadas as curvas alternativas apresentadas a seguir. Para possibilitar a inversão e aplicação de logaritmos, a variável foi modificada, considerando-se como 0.1 o valor mínimo (imóveis novos). Outras modificações semelhantes foram testadas, sem variações significativas nos modelos. 
Tabela A - Variáveis consideradas na análise de regressão*

\begin{tabular}{|c|c|c|c|}
\hline variável & tipo ** & unid & descrif̧ao \\
\hline VALOR & $\mathrm{C}$ & RS & $\begin{array}{l}\text { valor total, corrigido até janeiro de } 1996 \text { pelo } \\
\text { IGP-DI (FGV) }\end{array}$ \\
\hline ÁREA & $\mathrm{C}$ & $\mathbf{m}^{2}$ & área total construida \\
\hline BAIRRO & $\mathrm{C}$ & $\cdot$ & $\begin{array}{l}\text { classificação baseada na renda média por } \\
\text { bairro (BRASIL, 1980) }\end{array}$ \\
\hline DISTÂNCIA & c & km & $\begin{array}{l}\text { distância ao centro comercial/histórico da } \\
\text { cidade (CBD) }\end{array}$ \\
\hline GARAGEM & D & - & $\begin{array}{l}\text { indica a existência de garagem ou box de } \\
\text { estacionamento }\end{array}$ \\
\hline IDADE & C & ano & $\begin{array}{l}\text { idade fiscal da construģão (data do "Habite- } \\
\text { se") }\end{array}$ \\
\hline 105 & $\mathbf{D}$ & $\cdot$ & dummy para idade entre 0 e 5 anos \\
\hline 110 & $\mathbf{D}$ & $\cdot$ & dummy para idade entre 6 e 10 anos \\
\hline 115 & $\mathrm{D}$ & - & dummy para idade entre 11 e 15 anos \\
\hline 120 & D & $\cdot$ & dummy para idade entre 16 e 20 anos \\
\hline 125 & D & - & dummy para idade entre 21 e 25 anos \\
\hline 130 & D & - & $d u m m y$ para idade entre 26 e 30 anos \\
\hline 140 & $\mathbf{D}$ & $\cdot$ & dummy para idade entre 31 e 40 anos \\
\hline Iso & $\mathrm{D}$ & $\cdot$ & dummy para idade entre 41 e 50 anos \\
\hline LUXO & D & - & $\begin{array}{l}\text { dummy para imóveis especiais ( de padrão } \\
\text { superior) }\end{array}$ \\
\hline MES & $\mathrm{C}$ & mês & $\begin{array}{l}\text { número de meses desde janeiro de } 1990 \text { até a } \\
\text { transação }\end{array}$ \\
\hline
\end{tabular}

*er texto para esclarecimentos; ** Tipo: C-variável contínua; D-dicotômica 


\section{PRODUÇÃO}

A comparação e escolha da melhor curva pode ser feita pela estatística t, calculada para cada variável (ao nível de $95 \%$, tolerando-se até $90 \%$ ), pela estatística $F$, pelo coeficiente de determinação $\mathbf{R}^{2} \mathbf{a}$, ajustado para os graus de liberdade, e pelo erro padronizado (SE), calculados para cada modelo, na forma como geralmente são empregados nas análises de regressão. As equações são as descritas na Tabela 2. A variável Distância não foi significativa para nenhum dos modelos apresentados, provavelmente pelo caráter não monocêntrico da cidade. Todos os modelos foram aprovados nos testes estatísticos básicos. Em alguns, surgiram leves tendências nos residuos, toleradas devido ao caráter exploratório da análise. Os modelos foram mantidos semelhantes, tomando como base o linear (1), para facilitar a comparação. Percebe-se a estabilidade dos coeficientes e suas significâncias, nas variáveis que aparecem em todos os modelos. A variação nas constantes (interceptos) está relacionada com a diversidade dos formatos da variável Idade.

O conjunto de oito variáveis indicadoras (dummies $\underline{105} \sim \underline{\mathrm{I} 00}$ ) busca avaliar as peculiaridades de cada faixa de idade. Foi incluída a reta (Idade) como base, sendo incluidas apenas as dummies de coeficiente $\mathbf{t}$ em valores razoáveis (acima de 2). O modelo com estas variáveis é o sexto. Da análise do modelo, percebe-se que apenas os imóveis de idades entre 6 e 25 anos demonstraram a necessidade de valores diferentes da reta.
O modelo 6 é ligeiramente superior ao modelo 1, que empregava a reta, simplesmente.

Nos modelos 4 e 5 , a constante apresentou significância excessivamente baixa (29\% e $16 \%$ ), e foi removida (isto equivale a forçá-la a ter valor zero). Com esta providência, os coeficientes das variáveis explicativas praticamente não foram alterados, mas os modelos melhoraram em cerca de $10 \%$ no coeficiente de determinação (de $77 \%$ para $\mathbf{8 7 \%}$ ). Justamente estes dois modelos foram os de melhor desempenho geral (F, $R^{2} \mathbf{a}, S E$ ). A idade foi considerada com as transformações logarítmica e raiz quadrada, nestes modelos.

\section{Comparação dos modelos e métodos}

Os coeficientes que multiplicam a variável Idade permitem a comparação com as fórmulas tradicionais. Como exemplo, apresenta-se na Tabela 3 os efeitos da depreciação calculados para um apartamento de 14 anos de idade em relação a um idêntico, porém novo. A vida útil (usada nas fórmulas) foi considerada de 70 anos (este imóvel estaria em $20 \%$ de sua vida útil, portanto). As características empregadas nas equações foram as médias da amostra, valores para os quais a regressão apresenta a melhor predição, que são os indicados na Tabela 1 , exceto por: $B o x=0, L u x o=0$ e Idade $=14$ anos. 
PRODUÇÃO

Tabela B - Estatísticas para os modelos usando como variável dependente VALOR

\begin{tabular}{|c|c|c|c|c|c|c|c|c|c|c|c|c|}
\hline $\begin{array}{l}\text { modelo } \\
\text { variável }\end{array}$ & $\begin{array}{l}1 \\
\text { coef. }\end{array}$ & $t$ & $\begin{array}{l}2 \\
\text { coef. }\end{array}$ & $\mathbf{t}$ & $\begin{array}{l}3 \\
\text { coef. }\end{array}$ & t & $\begin{array}{l}4 \\
\text { coef. }\end{array}$ & $t$ & $\begin{array}{l}5 \\
\text { coef. }\end{array}$ & $\mathbf{t}$ & $\begin{array}{l}6 \\
\text { coef. }\end{array}$ & $\mathbf{t}$ \\
\hline Área & 364 & 36.9 & 373 & 39.0 & 374 & 39.0 & 361 & 38.0 & 359 & 37.0 & 358 & 36.8 \\
\hline Bairro & 1369 & 5.4 & 1089 & 4.5 & 1078 & 4.4 & 1213 & 7.5 & 1434 & 8.2 & 1179 & 4.7 \\
\hline Garagem & 16330 & 8.2 & 16877 & 8.7 & 17195 & 8.9 & 14926 & 7.8 & 15254 & 7.9 & 15393 & 7.9 \\
\hline Idade & -425 & -7.9 & - & & - & & - & & - & & -451 & -7.9 \\
\hline 1/Idade & - & & 1763 & 9.7 & - & & - & & - & & - & \\
\hline 1//dade ${ }^{2}$ & - & & . & & 166 & 9.2 & - & & - & & - & \\
\hline LnIdade & - & & - & & - & & -3751 & -11.5 & - & & - & \\
\hline Idade $^{1 / 2}$ & - & & - & & - & & - & & .3451 & -10.2 & - & \\
\hline 105 & - & & . & & - & & - & & - & & - & \\
\hline 110 & - & & - & & - & & - & & - & & -9387 & -6.2 \\
\hline 115 & - & & - & & - & & - & & - & & -7022 & -4.7 \\
\hline 120 & - & & - & & - & & - & & - & & -5370 & -3.0 \\
\hline I25 & - & & - & & - & & - & & - & & -4878 & -2.7 \\
\hline 130 & - & & . & & - & & - & & - & & - & \\
\hline 140 & - & & . & & - & & - & & - & & - & \\
\hline I50 & - & & - & & - & & - & & - & & - & \\
\hline Luxo & 98734 & 17.9 & 98150 & 18.0 & 98318 & 18.0 & 97286 & 17.5 & 97791 & 18.0 & 97696 & 18.0 \\
\hline LnMês & -2491 & -3.2 & -2716 & -3.6 & -2689 & 3.5 & -2910 & -5.0 & -2499 & -4.2 & -2975 & -3.9 \\
\hline constante & -5339 & -1.3 & -9489 & -2.3 & -9246 & -2.3 & 0 & * & 0 & * & 4159 & 1.0 \\
\hline$R^{2} a$ & \multicolumn{2}{|l|}{$72.78 \%$} & \multicolumn{2}{|l|}{$73.53 \%$} & \multicolumn{2}{|l|}{$73.31 \%$} & \multicolumn{2}{|l|}{$87.38 \%$} & \multicolumn{2}{|l|}{87.07} & \multicolumn{2}{|l|}{$73.85 \%$} \\
\hline F & \multicolumn{2}{|l|}{459.42} & \multicolumn{2}{|l|}{477.36} & \multicolumn{2}{|l|}{472.07} & \multicolumn{2}{|l|}{1190.10} & \multicolumn{2}{|l|}{1156.80} & \multicolumn{2}{|l|}{291.55} \\
\hline$S E$ & \multicolumn{2}{|l|}{17173} & \multicolumn{2}{|l|}{16934} & \multicolumn{2}{|l|}{17004} & \multicolumn{2}{|l|}{16659} & \multicolumn{2}{|l|}{16866} & \multicolumn{2}{|l|}{16832} \\
\hline
\end{tabular}

* A constante foi removida nestes modelos (ver texto). 


\section{PRODUÇÃO}

\section{Tabela C - Cálculo da depreciação pelos métodos e modelos apresentados}

\begin{tabular}{c|c|c|c}
\hline procedimento & valor novo (R\$) & valor depreciado (R\$) & $\begin{array}{c}\text { percentual } \\
\text { depreciado }\end{array}$ \\
\hline modelo 1 & 36999,01 & 31090,01 & $15,97 \%$ \\
modelo 2 & 46814,09 & 29310,02 & $37,39 \%$ \\
modelo 3 & 46058,85 & 29459,69 & $36,04 \%$ \\
modelo 4 & 47256,37 & 28720,27 & $39,22 \%$ \\
modelo 6 & 41690,33 & 29870,70 & $28,35 \%$ \\
\hline método da reta & 45000,00 & 28502,79 & $31,80 \%$ \\
método da & 45000,00 & 37800,00 & $16,00 \%$ \\
parábola & 45000,00 & 43560,00 & $3,20 \%$ \\
método de Ross & $40690 \%$ & $9,60 \%$ \\
\hline
\end{tabular}

A semelhança da depreciação calculada pelo modelo 1 e pelo método da reta é notável. Outros valores calculados também foram semelhantes, tornando-os equivalentes. Todavia, $o$ primeiro modelo é o de menor desempenho estatístico, podendo ser considerado inferior aos demais. Os modelos 4 e 5 , considerados os melhores, apresentam depreciações distintas. A depreciação pela equação 4 é aparentemente elevada, porém deve-se ao formato da curva logarítmica, que provoca efeito forte no início e suave no final. Calculando para 70 anos, por exemplo, a depreciação é de $52 \%$. Na verdade, esta curva amplia o valor residual (Vr).
As variações nos percentuais podem vir de peculiaridades dos dados empregados ou de problemas nas variáveis (erros de medida na variável que mede a localização, por exemplo). A análise mais detalhada poderá sugerir transformações distintas para as variáveis em cada modelo, ao contrário do formato único empregado aqui, variando apenas o formato da idade. De qualquer forma, trata-se de um estudo exploratório, mas as equações de regressão foram aprovadas pelos testes estatísticos e permitem supor depreciação na faixa de $28 \%$ a $39 \%$, muito superiores às calculadas pelas fórmulas. 


\section{Conclusão}

A utilização de coeficientes tabelados ou fórmulas padronizadas para descrever o mercado imobiliário não é aconselhável. Eventualmente, isso torna-se necessário, para comparação com modelos de outros profissionais, que adotaram estes métodos, ou por falta de dados para análise de regressão, como indicou Lima (1995), em seu contexto de análise.

Neste artigo descreve-se os procedimentos para investigação da curva de depreciação, através de uma amostra de apartamentos residenciais transacionados em Porto Alegre. Emprega-se regressão múltipla para verificação de cada opção, testando-se várias alternativas.

Dentre os modelos apresentados, as curvas de melhor ajuste foram as que consideraram o tempo (variável Idade) na forma logaritmica e com expoente $1 / 2$. Ressalta-se porém que trata-se de um ajuste empírico: os modelos apresentados são exemplos do procedimento de análise e não podem ser generalizados. Ao contrário, fica a sugestão para que análises semelhantes sejam realizadas em outras cidades, disseminando o processo e permitindo eventualmente a comparação das curvas e investigação de um padrão de similaridades ou dissimilaridades na depreciação de imóveis urbanos.

Recebido em: 10/11/97 Aceito em: 15/06/98

Publicado em: 27/07/98

\section{Bibliografia}

ABNT (Associação Brasileira de Normas Técnicas). Avaliações de imóveis urbanos (NB 502). Rio de Janeiro: ABNT, 1989.

ABRAMO, Pedro. A dinâmica imobiliária: elementos para o entendimento da espacialidade urbana. Rio de Janeiro: IPPUR/UFRJ, 1988. Dissertação (Mestrado em Planejamento Urbano).

BALARINE, Oscar Fernando Osório. Determinação do impacto de fatores sócio-econômicos na formação do estoque habitacional em Porto Alegre. Porto Alegre: Ed.PUCRS, 1996.

BRASIL. Censos demográficos. Rio de Janeiro: IBGE, 1980.

CUTHBERTSON, Keith; HALL, Stephen G.; TAYLOR, Mark P. Applied econometric techniques. New York: Harvester Wheatsheaf, 1992.

DUBIN, Robin A. Spatial autocorrelation and neighborhood quality. Regional Science and Urban Economics, v.22, n.3, p.433-452, Sept. 1992.

FIKER, José. Avaliação de terrenos e imóveis urbanos. 4ed. São Paulo: PINI, 1993.

FRANCHI, Cláudia de Césare. Avaliação das características que 


\section{PRODUÇÃO}

contribuem para a formação do valor de apartamentos na cidade de Porto Alegre. Porto Alegre: UFRGS, 1991. Dissertação (Mestrado em Engenharia).

GONZÁLEZ, Marco Aurélio Stumpf. A formação do valor de alugueis de apartamentos residenciais na cidade de Porto Alegre. Porto Alegre: UFRGS, 1993. Dissertação (Mestrado em Engenharia).

- Planta inferencial de valores com dados de ITBI. Porto Alegre: NORIE/UFRGS, 1996.

A engenharia de avaliações sob a ótica inferencial. São Leopoldo: Ed. UNISINOS, 1997. . Noções sobre o conceito de valor no mercado imobiliário. Anais IX COBREAP (Congresso Brasileiro de Engenharia de Avaliações e Perícias). São Paulo: IBAPE, p.143-149, 1997a.

IBAPE (Instituto Brasileiro de Avaliações e Perícias de Engenharia). Engenharia de avaliações. São Paulo: Pini, 1974.

LIMA, Gilson Pereira de Andrade. Homogeneizaçâo fundamentada - Uma utopia? Anais - VIII COBREAP (Congresso Brasileiro de Engenharia de Avaliações e Perícias). Florianópolis: ICAPE, p.303-314, Nov. 1995.

LOPES, José Tarcísio Doubek. Depreciação de edificações. Anais - VIII
COBREAP (Congresso Brasileiro de Engenharia de Avaliações e Perícias). Florianópolis: ICAPE, p.277-284, Nov. 1995.

LUCENA, José Mário Pereira de. $\mathbf{0}$ mercado habitacional no Brasil. Rio de Janeiro: FGV, 1985.

MARASCHIN, Clarice. Alterações provocadas pelo shopping center em aspectos da estrutura urbana Iguatemi, Porto Alegre, RS. Porto Alegre: PROPUR/UFRGS, 1993. Dissertação (Mestrado em Planejamento Urbano).

McMICHAEL, Stanley L. McMichael's appraising manual. 4ed. Englewood Cliffs (NJ): Prentice-Hall, 1962.

MOREIRA, Alberto Lélio. Princípios de engenharia de avaliações. 3ed. São Paulo: PINI, 1994. 